

CHALMERS

UNIVERSITY OF TECHNOLOGY

\title{
Intersymbol interference in DQPSK fibre-optic systems
}

Downloaded from: https://research.chalmers.se, 2023-04-26 09:53 UTC

Citation for the original published paper (version of record):

Zhao, H., Agrell, E., Karlsson, M. (2009). Intersymbol interference in DQPSK fibre-optic systems. European Transactions on Telecommunications, 20(8): 758-769. http://dx.doi.org/10.1002/ett.1354

N.B. When citing this work, cite the original published paper. 


\title{
Intersymbol Interference in DQPSK Fiber-Optic Systems
}

\author{
Hongxia Zhao ${ }^{\dagger}$, Erik Agrell ${ }^{\dagger}$, Magnus Karlsson ${ }^{\ddagger}$ \\ ${ }^{\dagger}$ Department of Signals and Systems, Chalmers University of Technology, SE-412 96 Göteborg, Sweden. E-mail: hz,agrell@chalmers.se \\ ${ }^{\ddagger}$ Department of Microtechnology and Nanoscience, Chalmers University of Technology, SE-412 96 Göteborg, Sweden.
}

\begin{abstract}
The limited bandwidth of high-speed transmitter and receiver hardware gives rise to intersymbol interference (ISI) in digital communication systems. The influence of such ISI is investigated in a low-complexity fiber-optical setting, using differential quaternary phase shift keying (DQPSK) modulation. The ISI is approximated by a memoryless, stochastic model, for use in applications where neither equalization nor sequence detection can be afforded. The channel capacity of this model is calculated and shown to depend strongly on the transmitter calibration. More than $3 \mathrm{~dB}$ is gained, at a target bit error rate of $10^{-6}$, by allowing the transmitted phase levels to deviate from their nominal multiples of $\pi / 2$ by up to $7 \%$.
\end{abstract}

\section{INTRODUCTION}

In high-speed fiber-optical communication systems, the nonzero rise time of electrooptical modulators deteriorates the system performance. In combination with the receiver lowpass filters, which are also nonideal, the nonideal transmitters introduce intersymbol interference (ISI) to the demodulated signal, even at short ranges where the fiber dispersion is insignificant. In a previous paper [1], we analyzed the influence of nonideal hardware on coherent quaternary phaseshift keying (QPSK) transmission in a fiber-optical system. We showed that the ISI, if not compensated for, causes unequal bit error rates (BER) between the two QPSK bit streams and an overall performance degradation.

The work in [1] is here extended to differential QPSK (DQPSK) systems. The ISI that is visible in PSK constellations and found to influence the two bits in the QPSK symbol differently is averaged over all transmitted phases for DQPSK, and is thus not visible as a BER difference between the two DQPSK bits. However, the effect is still present as a limitation in DQPSK systems. As will be shown, it causes unequal symbol error rates (SER), conditioned on the transmitted symbol, and it increases the average BER. We also discuss and quantify how simple modifications to the transmitter can improve the system performance.

From a communication-theoretical perspective, the differences between QPSK and DQPSK are significantly altered noise statistics and the requirement for differential precoding. From a more practical fiber communication perspective, the DQPSK receiver is significantly easier and less complex to realize than the coherent receiver required for QPSK, although it comes at the expense of an SNR penalty. In fact, optical DQPSK systems are significantly more mature and closer to commercialization than coherent QPSK systems, which is a result of having been in the research focus for the last 6-8 years. Indeed, the current transmission record over a single fiber $(25 \mathrm{~Tb} / \mathrm{s})$ was enabled by DQPSK modulation [2]. It is of crucial importance for system designers, as well as telecom operators, to fully understand the cost/complexity vs. performance trade-offs of DQPSK and QPSK systems. We will return to this trade-off discussion when discussing the optical transmitters in Section II-A.

DQPSK modulation is well understood theoretically for additive Gaussian noise and ideal transmitters and receivers [3, Sec. 5.2.8]. It is used in numerous wireless standards (such as the IEEE 802.11 standards for wireless LAN) and there is now a rapidly growing interest in using DQPSK modulation also in fiber-optical communications. The performance of such systems was theoretically analyzed in, e.g., [4], [5] and references therein. Experimental realizations of DQPSK systems were discussed in [6]-[9], and more recently in WDM configurations [2], [10], [11]. Effects of the optical transmitter and receiver on system performance and channel capacity have however never been studied theoretically.

The standard method to combat ISI is by equalization or sequence estimation [3, Ch. 10-11]. In a fiber-optical differential PSK setting, ISI has been discussed and mitigated, e.g., by skewed precoding [12], convolutional error-correction codes [13], symmetric DPSK [14], electronic processing [15], [16], and maximum liklihood sequence estimation [17]. In those papers, the main ISI source is fiber dispersion and receiver filtering, while ISI from transmitter imperfections was studied to a less extent.

In this work, we consider a scenario where equalization or sequence estimation is too complex. The question is, can this type of ISI be mitigated to some degree, without adding any additional hardware or processing resources? The answer is yes, by up to $3 \mathrm{~dB}$. We show this by developing a stochastic model for the ISI, calculating the theoretical channel capacity of the model, finding transmitter parameters that maximize the capacity, and simulating the BER using this modified transmitter.

\section{System Model}

The dominating impairment in the studied communication system is, apart from the nonideal hardware, the amplified spontaneous emission noise of the erbium-doped fiber amplifier (EDFA). Other mitigations like thermal noise, shot noise, 
and all kinds of dispersion and nonlinear effects are ignored. The data rate is $20 \mathrm{Gbit} / \mathrm{s}$ (10 Gsymbol/s).

\section{A. Transmitter}

At bit rates of $10 \mathrm{~Gb} / \mathrm{s}$ or higher, the commonly used transmitter is based on an ideal continuous wave (CW) laser and an external modulator [18, p. 122]. In short, the transmitter converts the $\mathrm{CW}$ light radiated from the laser into a datacoded pulse train with the proper modulation format. To transmit a DQPSK signal, different transmitters with varying complexities have been investigated [19]-[21]. In [19], three DQPSK transmitters are compared. The two simplest transmitters in [19] are used in this paper and are denoted as TX-A and TX-B. TX-A uses two modulators, one amplitude modulator and one phase modulator, which are connected serially, while TX-B uses a single phase modulator [1]. These transmitter configurations are the simplest from a cost/complexity perspective. It is well known that e.g. the parallel Mach-Zehnder modulator (MZM) may give better performance, partly from the forgiving electrical-to-optical transfer characteristics that reduce the influence of noise from the electric driving signals. From a system vendor perspective, these modulators have significant drawbacks such as requiring active biasing and temperature stabilization, besides being intrinsically more costly and less mature especially at high bandwidths ( $>40$ $\mathrm{GHz}$ ) [22]. Therefore the parallel MZM, being common in research, is by no means a commodity in system development.

Alternatively, a pulsed DQPSK (often denoted (RZDQPSK) transmitter could be used, requiring the use of an additional modulator for pulse carving or a mode-locked laser source. While this solution has less transmitter ISI, it is also more com plex and costly, and requires more bandwidth which is a drawback in wavelength division demultiplexing systems. We therefore believe that there is a strong interest, not least from optical system designers, to evaluate the ultimate performance of these simpler, low-cost transmitter structures.

The normalized baseband transmitted signals of TX-A and TX-B, denoted as $f_{A}(t)$ and $f_{B}(t)$, respectively, are

$$
f_{A}(t)=\eta \cdot \sin \left[\frac{\pi}{2} \sum_{n}\left(a_{n} p(t-n T)\right)\right] e^{j \frac{\pi}{2} \sum_{n} b_{n} p(t-n T)},
$$

and

$$
f_{B}(t)=\eta \cdot e^{j \frac{\pi}{2} \sum_{n}\left(a_{n}+b_{n}\right) p(t-n T)},
$$

where $\eta$ is a power control scaling factor, $T$ is the symbol time, $a_{n}$ and $b_{n}$ are the binary output of mapping units that map to the DQPSK coded bits, and $p(t)$ is the impulse response of a pulse shaper. The pulse shaper is used to model the limited temporal response of the transmitter, and it has a unity max raised cosine (RC) impulse response in the time domain. The roll-off factor of the RC pulse is determined by the bandwidth of the hardware electronics, i.e., the higher the transmission data rate, the larger the roll-off factor because of the bandwidth limitation of the electronics. In previous works, it has been set to 0.5 [19], or 0.4 and 1 [23]. In this paper, a value of 0.7 is used, considering the moderate transmission rate. Further details of $p(t)$ can be found in [1].

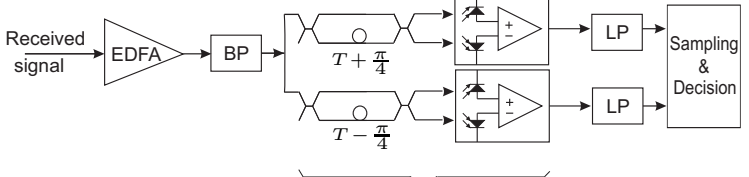

Differential detector

Fig. 1. Structure of the system receiver.

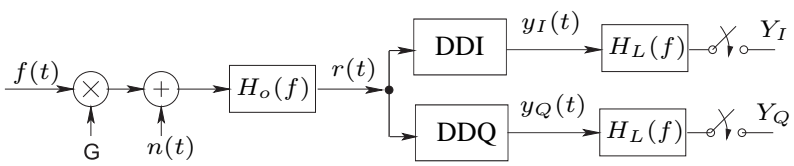

Fig. 2. Equivalent system baseband model.

Since the transmitted bits modulated by the phase modulator based transmitter are naturally mapped, a DQPSK precoder is needed, not only to realize the differential encoding but also the Gray mapping of the information bits. This precoder is thus more complex compared to what is used in digital wireless communications. The logic operations of the precoder used in this paper are [24]

$$
\begin{gathered}
c_{1, n}=\left(\left(\left(d_{1, n} \oplus d_{2, n}\right) \wedge c_{2, n-1}\right) \oplus d_{1, n}\right) \oplus c_{1, n-1} \\
c_{2, n}=\left(d_{1, n} \oplus d_{2, n}\right) \oplus c_{2, n-1}
\end{gathered}
$$

where $n$ stands for the index of the binary streams, $d_{i, n}$ and $c_{i, n}$ for $i \in\{1,2\}$ denote the information bits and the coded bits, $\oplus$ and $\wedge$ are exclusive or (XOR) and the AND operation, respectively. The precoder outputs, the coded bits $c_{1, n}$ and $c_{2, n}$, then pass through a mapping unit and map to $a_{n}$ and $b_{n}$, resp., as $a_{n} \in\{-1,1\}$ and $b_{n} \in\{0,1\}$.

\section{B. Receiver}

In the system receiver (see Fig. 1), the received signal is first amplified by an EDFA before passing through an optical bandpass (BP) filter. The output of the BP filter is split into two branches, subsequently referred to as the inphase (I) and quadrature (Q) branch. In each branch, the signal is first differentially detected, then filtered by an electrical lowpass (LP) filter. Finally, a sampling and decision unit makes a decision based on the outputs of the two branches. Note that there is no equalizer in this system. The purpose of this paper is to demonstrate that the adverse effects of ISI in optical communications can be reduced without increasing the complexity of the receiver. Hence, equalization and sequence estimation, which require the implementation of high-speed A/D conversion and digital signal processing, is beyond the scope of this study. We refer to [25]-[27] for results of sequence estimation and equalization in fiber-optic receivers.

A model of the receiver is created to simplify our study (Fig. 2). In the model, $G, n(t), H_{o}(f)$ and $H_{L}(f)$ represent the the EDFA gain, the optical noise and the frequency responses (low-pass equivalent) of the BP and LP filters, respectively. The DDI/DDQ denote the differential detector inphase (DDI)/quadrature (DDQ) branch. The differential detector includes a delay interferometer with a differential delay 


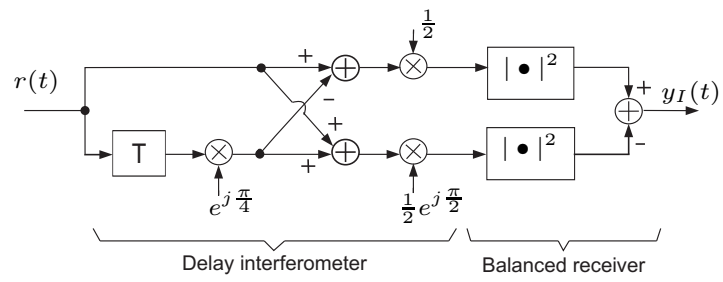

Fig. 3. Equivalent differential detector I (DDI) branch.

of one symbol $\pm \pi / 4$ and a balanced receiver, which comprises two photodiodes [28]. Fig. 3 shows the mathematical diagram of the differential detector I branch, where $\mathrm{T}$ represents the symbol time, and the square function $|\cdot|^{2}$ is due to the photo diode, which is assumed to have a unit responsivity. This assumption is for notation simplicity only and will not affect the conclusions.

An EDFA not only amplifies the signal, it also contributes some noise $n(t)$. This noise is commonly modelled as a zeromean additive white Gaussian noise (AWGN) with singlesided noise power spectral density $N_{0}=\frac{F}{2} h \nu G$, where $F$ is the amplifier noise figure and $h \nu$ is the photon energy. Although the optimum bandwidth of the optical BP filter is around 2-3 times the data rate depending on the modulation format, the transmitter characteristics [23], [29], a sufficiently stable narrow-band optical filter is not a realistic solution. The BP filter in this paper is chosen to have a $3 \mathrm{~dB}$ bandwidth of $40 \mathrm{GHz}$ and a Gaussian frequency response. The equivalent baseband frequency response of the BP filter is

$$
H_{o}(f)=e^{-\frac{2 \ln 2 \cdot f^{2}}{B^{2}}},
$$

where $B$ is the $3 \mathrm{~dB}$ bandwidth.

The design of the low-pass filters, which are realized on the electrical side of the receiver, offers more flexibility than the bandpass filter. Because of the ISI existing in the transmitted signal due to the non-zero roll-off factor of the pulse shaper, and the nonlinear characteristics of the photo diodes, full optimization of the filter responses is a formidable problem in itself [30] and beyond the scope of this paper. In this paper, a Butterworth filter is chosen, which is commercially available and commonly used (see e.g. [31]). The frequency response of a Butterworth filter is

$$
H_{L}(f)=\sqrt{\frac{1}{1+\left(f / f_{c}\right)^{2 N}}},
$$

where $N$ is the order of the filter and $f_{c}$ is the cutoff frequency.

The decision, made from the output of I and Q branches, $Y_{I}$ and $Y_{Q}$, defines the received bits of the DQPSK symbol, which should, in the absence of transmission errors, recover the information bits $d_{1}$ and $d_{2}$.

\section{Simulation AND SER Results}

In all the simulations, random independent information bits with a total bit rate of $20 \mathrm{Gbps}$ are used, and the symbol error rate was estimated after more than 50-100 symbol errors had occurred.

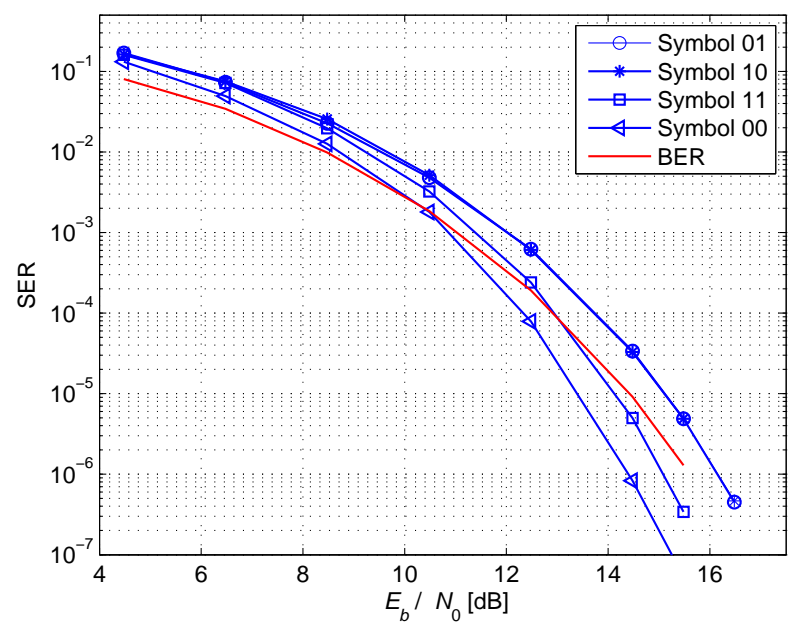

Fig. 4. Simulated symbol error rates when using TX-A.

At the receiver, the gain and the noise figure of the EDFA are set at $30 \mathrm{~dB}$ and $5 \mathrm{~dB}$ respectively. The carrier wavelength is $1550 \mathrm{~nm}$ and the bandwidth of the optical BP filter is 40 GHz. The Butterworth low-pass filters have an order of $N=3$ and a cutoff frequency of $f_{c}=7 \mathrm{GHz}$ in all simulations [29], [32].

When using transmitters TX-A and TX-B, the simulated SER, which is conditioned on each of the four transmitted symbols, versus $E_{b} / N_{0}$ are plotted in Figures 4 and 5, respectively. The $E_{b} / N_{0}$ is the ratio between bit energy and singlesided noise spectral density before the optical BP filter, and $E_{b}$ is calculated as $E_{b}=\frac{1}{2} E_{s}$ and $E_{s}=G^{2} \int_{t_{0}}^{t_{0}+T}|f(t)|^{2} d t$, where $E_{s}$ is the symbol energy ${ }^{1}, T$ is the symbol time, $t_{0}$ is an arbitrary time instant and $f(t)$ is the received signal. The symbols, $\{00,01,10,11\}$, shown in the figures represent the two information bits as $\left\{d_{1} d_{2}\right\}$. Since TX-B has only one modulator while TX-A consists of two modulators, TXB has a simpler structure. It is thus no surprise to see that the system using TX-A performs better than the system using TX-B, which has been reported in [19]. For comparison, the average BER of the system is also included in the figures.

As seen in the figures, symbol 00 performs the best for both systems. When using TX-A, symbols 01 and 10 have similar performance and are worse than symbol 11. However, when using TX-B, symbol 11 performs the worst and gives almost twice the error rate of symbols 01 and 10. Again these two symbols perform similarly and much worse than symbol 00 . The performance differences between the best and the worst symbols are about $2 \mathrm{~dB}$ and $4 \mathrm{~dB}$ at a SER of $10^{-6}$ when using TX-A and TX-B, respectively. This difference is even larger if the target SER is lower. The two bits of the DQPSK symbol have equal BER performance, which is different from a coherent system where the two QPSK bits performs differently [1]. The reason why unequal SERs give equal BER performances is that the symbol performances are

\footnotetext{
${ }^{1}$ When using TX-A, $E_{s}$ is averaged over a large number of symbols since the energy between symbols is slightly different due to the use of amplitude modulator.
} 


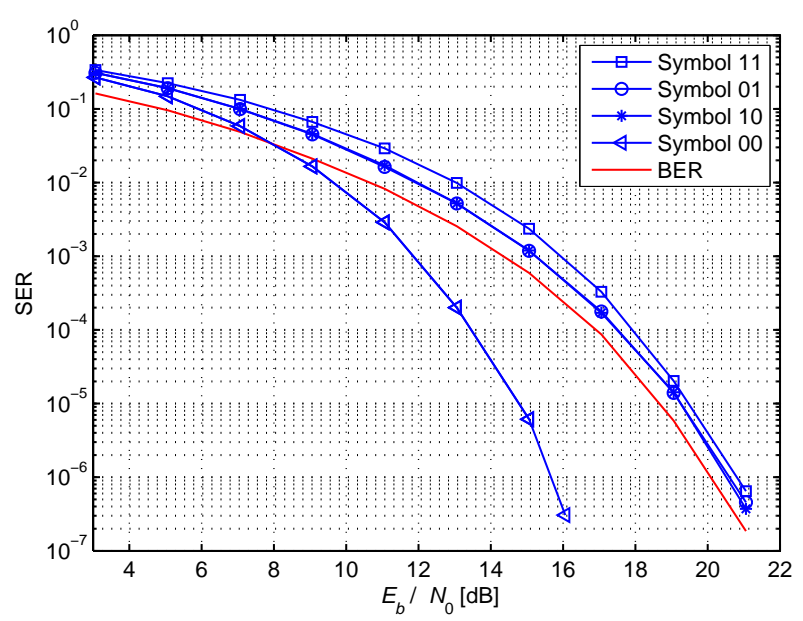

Fig. 5. Simulated symbol error rates when using TX-B.
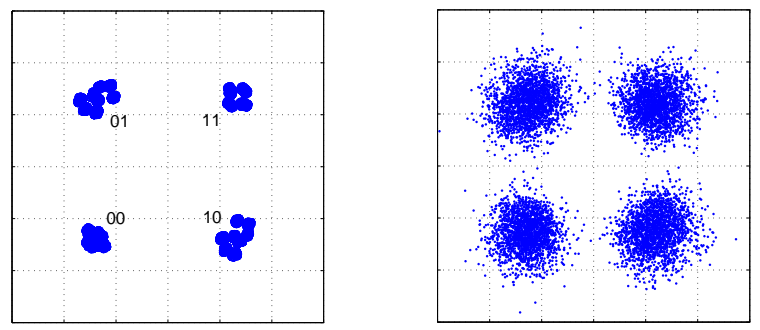

Fig. 6. Simulated signal constellation $\left(Y_{I}, Y_{Q}\right)$ without noise (left) and with noise (right) when using TX-A, $E_{b} / N_{0}=13 \mathrm{~dB}$ when with noise.

averaged when computing BERs.

To illustrate why the unequal SER occurs, we plot the signal constellation of the sampled low-pass filter output $\left(Y_{I}, Y_{Q}\right)$ in Figures 6 and 7, when systems using TX-A and TX-B, respectively. The bits of the symbols, $\{00,01,10,11\}$, shown in the figures represent the information bits $\left\{d_{1} d_{2}\right\}$. From the figures, it can be easily seen that the four symbol clouds are asymmetric, especially in Fig. 7 where the system uses TX-B. A more dislocated symbol cloud means that there are more signal points close to the decision boundaries, which are the $\mathrm{x}$ and $y$ axes in this case. Since the signal points which are close to the boundaries dominate the performance at a high signalto-noise ratio (SNR), the asymmetric signal constellation will lead to the unequal symbol performance. When TX-A is used, symbols 00 and 11 have less dislocated clouds than symbols 10 and 01 , and they thus have better performance. However, when using TX-B, symbol 11 has the worst performance, the reason being that it has the most dislocated cloud.

\section{TheORETICAL SER RESUlTS}

In this section, the theoretical symbol error rates of the studied DQPSK systems are derived based on the system model described in Sec. II. We assume that the transmitter and receiver together have a finite memory of $m$ symbols and the ISI only occurs in $m=4$ consecutive symbols in our derivation. This assumption is accurate at a reasonable noise level.
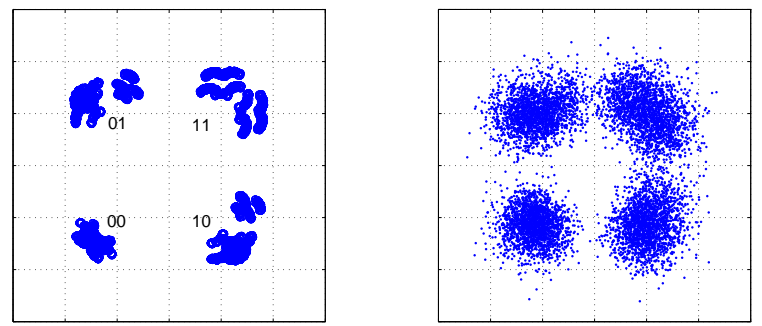

Fig. 7. Simulated signal constellation $\left(Y_{I}, Y_{Q}\right)$ without noise (left) and with noise (right) when using TX-B, $E_{b} / N_{0}=13 \mathrm{~dB}$ when with noise.

With an ideal fiber as assumed in Sec. II, the signal $f(t)$ in Fig. 2 equals either $f_{A}(t)$ (when using TX-A) or $f_{B}(t)$ (when using TX-B). From the figure, we can write signal $r(t)$ as $r(t)=(G f(t)+n(t)) * h_{o}(t)$, where the asterisk denotes convolution and $h_{o}(t)$ is the BP filter impulse response. Since the bandwidth of the received signal $f(t)$ is much smaller than the BP filter bandwidth, it is reasonable to assume that the signal $f(t)$ is undistorted after the BP filter such that $r(t)=$ $G f(t)+\hat{n}(t)$, where $\hat{n}(t)$ is the noise $n(t)$ filtered by the BP filter $H_{o}(f)$. For derivation convenience, we further rewrite the complex signal $r(t)$ as $r(t)=A_{s}(t) e^{-j \phi(t)}+N_{s}(t) e^{-j \theta(t)}$, such that $A_{s}(t) e^{-j \phi(t)}=G f(t)$ and $N_{s}(t) e^{-j \theta(t)}=\hat{n}(t)$. The outputs of the DDI and DDQ in Fig. 3 then can be derived as:

$$
\begin{aligned}
y_{I}(t)= & -A_{s}(t) A_{s}(t-T) \cos \left(\phi(t)+\frac{\pi}{4}-\phi(t-T)\right) \\
- & A_{s}(t) N_{s}(t-T) \cos \left(\phi(t)+\frac{\pi}{4}-\theta(t-T)\right) \\
- & N_{s}(t) A_{s}(t-T) \cos \left(\theta(t)+\frac{\pi}{4}-\phi(t-T)\right) \\
& -N_{s}(t) N_{s}(t-T) \cos \left(\theta(t)+\frac{\pi}{4}-\theta(t-T)\right), \\
y_{Q}(t)= & -A_{s}(t) A_{s}(t-T) \cos \left(\phi(t)-\frac{\pi}{4}-\phi(t-T)\right) \\
& -A_{s}(t) N_{s}(t-T) \cos \left(\phi(t)-\frac{\pi}{4}-\theta(t-T)\right) \\
& -N_{s}(t) A_{s}(t-T) \cos \left(\theta(t)-\frac{\pi}{4}-\phi(t-T)\right) \\
& -N_{s}(t) N_{s}(t-T) \cos \left(\theta(t)-\frac{\pi}{4}-\theta(t-T)\right) .
\end{aligned}
$$

The first terms of the equations present the desired signal while the other three terms are the noises. To calculate the SER, we write the sampled LP filter outputs as $Y_{I}=S_{I}+N_{I}$ and $Y_{Q}=S_{Q}+N_{Q}$, where $S_{I}$ and $S_{Q}$ are the desired signals and $N_{I}$ and $N_{Q}$ are the noises. The desired signal parts, $S_{I}$ and $S_{Q}$, are the convolutions of the LP filter impulse response with the signal components of $y_{I}(t)$ and $y_{Q}(t)$, respectively. For the Butterworth filter of order 3 with the frequency response given by (4), the impulse response is

$$
\begin{aligned}
h_{L}(t)= & 2 \pi f_{c}\left(e^{-2 \pi f_{c} t}-e^{-\pi f_{c} t} \cos \left(\sqrt{3} \pi f_{c} t\right)\right. \\
& \left.+\frac{1}{\sqrt{3}} e^{-\pi f_{c} t} \sin \left(\sqrt{3} \pi f_{c} t\right)\right), t \geqslant 0 .
\end{aligned}
$$

Since the transmitting adjacent NRZ pulses overlap, interferences come from both the previous and subsequent symbols. 

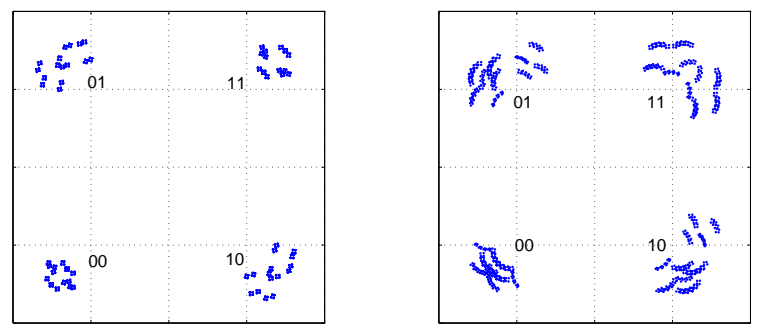

Fig. 8. Analytical signal constellations $\left(S_{I}, S_{Q}\right)$ when using TX-A (left) and TX-B (right).

In order to account for all the interference, with $m$ consecutive symbols included in $y_{I}(t)$ and $y_{Q}(t)$, the $(m-1)$ th symbol is set to be the desired one. Thus, $S_{I}$ and $S_{Q}$ are obtained from the convolution of $h_{L}(t)$ and the signal components of $y_{I}(t)$ and $y_{Q}(t)$ over $m-1$ symbol intervals,

$$
\begin{gathered}
S_{I}=\int_{\rho T}^{(m-1+\rho) T} y_{I}^{\Delta}(\tau) \cdot h_{L}((m-1) T-\tau) d \tau, \\
S_{Q}=\int_{\beta T}^{(m-1+\beta) T} y_{Q}^{\Delta}(\tau) \cdot h_{L}((m-1) T-\tau) d \tau,
\end{gathered}
$$

where $y_{I}^{\Delta}(\tau)$ and $y_{Q}^{\Delta}(\tau)$ are the first terms of (5)and (6), $0 \leq \rho \leq 0.7,0 \leq \beta \leq 0.7$, and their values are chosen by numerically optimizing the SER performance. The analytic signal constellation $\left(S_{I}, S_{Q}\right)$, which is equivalent to $\left(Y_{I}, Y_{Q}\right)$ without noise, is plotted in Fig. 8, and they agree very well with the simulations results shown in Figures 6 and 7. In the figure, each symbol has $4^{m}$ samples and in total $4^{m+1}$ samples are generated. The reason is that $f(t)$ needs to cover $m+1$ symbol intervals in order to have $m$ consecutive symbols in $y_{I}(t)$ and $y_{Q}(t)$ due to the differential decoding.

The noises $N_{I}$ and $N_{Q}$ are derived from the noise terms in (5) and (6). Since the last terms of the equations, $N_{s}(t) N_{s}(t-T) \cos \left(\theta(t) \pm \frac{\pi}{4}-\theta(t-T)\right)$, are relatively small compared to the dominant noises of terms 2 and 3 at relevant SNRs, it is ignored in our calculation. Because the signal amplitude and phase, $A_{s}(t)$ and $\phi(t)$, in the relevant noise terms are deterministic values at any given time, the noise statistic depends only on $N_{s}(t)$ and $\theta(t)$. Recalling that $N_{s}(t) e^{-j \theta(t)}=\hat{n}(t)$ and $\hat{n}(t)=n(t) * h_{o}(t)$, where $n(t)$ is an AWGN, it can be shown that $\hat{n}(t)$ is a zero-mean Gaussian process and the correlation of $\hat{n}(t)$ and $\hat{n}(t-T)$ can be ignored [33], [34]. The noise contributions in $y_{I}(t)$ and $y_{Q}(t)$ are thus zero-mean Gaussian processes. In particular, the noise samples at time $t=n T$ are Gaussian random variables with zero mean and variance of $\frac{N_{0}}{2} \cdot G^{2} \cdot \int_{-\infty}^{\infty}\left|H_{o}(f)\right|^{2} d f$ due to that $A_{s}(n T)=G$. Passing this zero-mean Gaussian process through a linear LP filter, the output is another zeromean Gaussian process in which each sample is a zero-mean Gaussian random variable. At time $t=n T$, the variance of those variables can be calculated as [3, Sec. 2.2.3]

$$
\sigma^{2}=\frac{N_{0}}{2} \cdot G^{2} \cdot \int_{-\infty}^{\infty}\left|H_{o}(f)\right|^{2}\left|H_{L}(f)\right|^{2} d f .
$$

With the low-complexity receiver studied in this paper, there are no means to exploit constructively the time diversity caused by ISI. Neither equalization nor sequence estimation is available, see Section II-B. A memoryless discrete-time model is therefore used for the channel, where the ISI is regarded as an additive non-Gaussian noise source. For ease of notation, we use $a$ to represent a symbol, $a \in \mathcal{A}=\{00,01,10,11\}$. Basically, the theoretical constellation clouds of symbol a is represented by the triplets $\left(S_{I}^{a}(i), S_{Q}^{a}(i), P^{a}(i)\right)$ for $i=$ $1,2, \cdots, 4^{m}$, where $P^{a}(i)$ is the probability of each point in the cloud of symbol $a$ and $\sum_{i=1}^{4^{m}} P^{a}(i)=1$ for all $a \in \mathcal{A}$. Using $X$ and $Y$ to represent the channel input and output, the transition probabilities are

$$
\begin{aligned}
& P\left(Y_{00} \mid X_{a}\right)=\sum_{i=1}^{4^{m}} P^{a}(i) Q\left(\frac{S_{I}^{a}(i)}{\sigma}\right) Q\left(\frac{S_{Q}^{a}(i)}{\sigma}\right), \forall a \in \mathcal{A} \\
& P\left(Y_{01} \mid X_{a}\right)=\sum_{i=1}^{4^{m}} P^{a}(i) Q\left(\frac{S_{I}^{a}(i)}{\sigma}\right) Q\left(\frac{-S_{Q}^{a}(i)}{\sigma}\right), \forall a \in \mathcal{A} \\
& P\left(Y_{10} \mid X_{a}\right)=\sum_{i=1}^{4^{m}} P^{a}(i) Q\left(\frac{-S_{I}^{a}(i)}{\sigma}\right) Q\left(\frac{S_{Q}^{a}(i)}{\sigma}\right), \forall a \in \mathcal{A} \\
& P\left(Y_{11} \mid X_{a}\right)=\sum_{i=1}^{4^{m}} P^{a}(i) Q\left(\frac{-S_{I}^{a}(i)}{\sigma}\right) Q\left(\frac{-S_{Q}^{a}(i)}{\sigma}\right), \forall a \in \mathcal{A}
\end{aligned}
$$

where $Q(x)=\frac{1}{\sqrt{2 \pi}} \int_{x}^{\infty} e^{-t^{2} / 2} d t$. The conditional symbol error rate can then be derived as

$$
\begin{aligned}
P_{e}\left(X_{a}\right)= & \sum_{\substack{b \in \mathcal{A} \\
b \neq a}} P\left(Y_{b} \mid X_{a}\right) \\
= & \sum_{i=1}^{4^{m}} P^{a}(i)\left[Q\left(\frac{\left|S_{I}^{a}(i)\right|}{\sigma}\right)+Q\left(\frac{\left|S_{Q}^{a}(i)\right|}{\sigma}\right)\right. \\
& \left.-Q\left(\frac{\left|S_{I}^{a}(i)\right|}{\sigma}\right) \cdot Q\left(\frac{\left|S_{Q}^{a}(i)\right|}{\sigma}\right)\right] .
\end{aligned}
$$

In Figures 9 and 10, the theoretical conditional SER are compared with the simulation results when using TX-A and TX-B, respectively. In all simulations, because of the use of random information bits, the a-priori probability is uniform distributed, $P\left(X_{a}\right)=1 / 4$. Thus, the uniform distributed a-priori probability is applied when computing (11). I.e., $P^{a}(i)=1 / 4^{m}$ for $i=1,2, \cdots, 4^{m}$. The analytical results are quite close to the simulations, especially when TX-A is used. When using TX-B, there are slight differences between analysis and simulation results at high SNRs. The reason is that the system using TX-B has more dislocated signal constellations and it is therefore more sensitive to the assumptions and approximations or their combinations ${ }^{2}$.

\section{Channel CAPACity}

As stated in Section II-B, the studied DQPSK systems use low-complexity receivers in which neither equalizations nor sequence detections are applied. The interference from other symbols are counted as additional noise when computing channel transition probabilities as in (10), the channels of

\footnotetext{
${ }^{2}$ These assumptions and approximations include that the ISI is only within $m$ symbol intervals, the BP filter has no effect on the transmitted signals and the noise is approximated to be Gaussian distributed.
} 


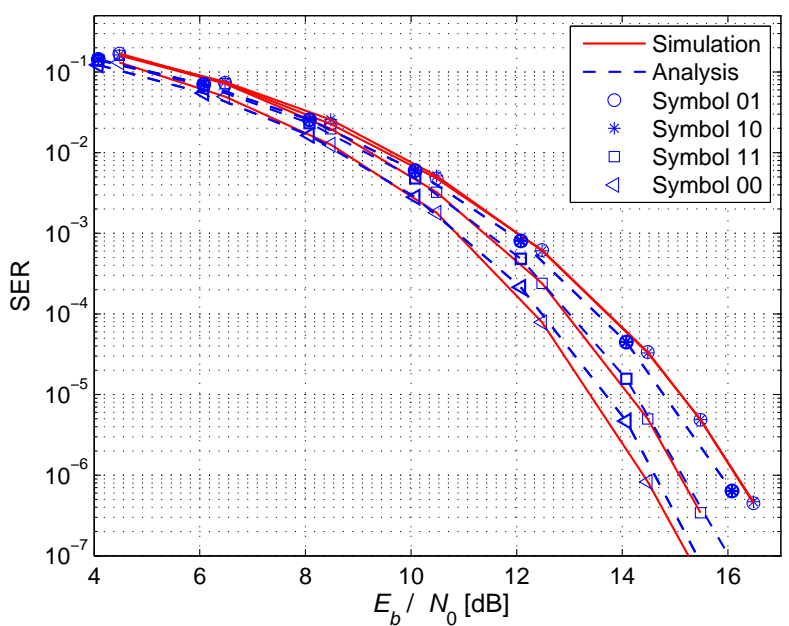

Fig. 9. Comparison of the simulated and analytical SERs when using TX-A.

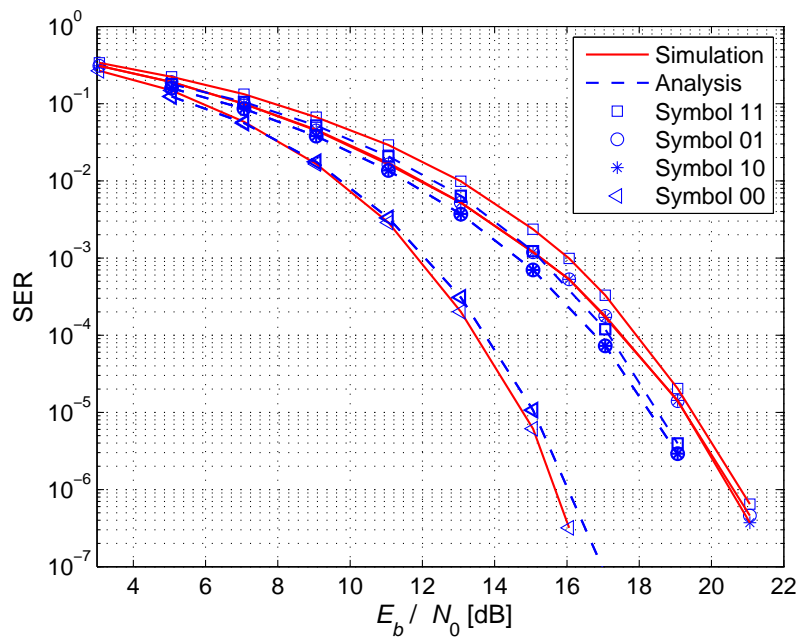

Fig. 10. Comparison of the simulated and analytical SERs when using TX-B.

such systems thus are memoryless. For memoryless channels, channel capacities are obtained by maximizing channel mutual information, which are related with a-priori and channel transition probabilities. In this section, two modifications are proposed in order to improve channel mutual information; one for a-priori probabilities and one for channel transition probabilities.

Given the transition probabilities $P\left(Y_{b} \mid X_{a}\right), \forall a, b \in \mathcal{A}$, the mutual information of the studied channel is defined as [3, pp. 382]

$$
I(X ; Y)=\sum_{a \in \mathcal{A}} \sum_{b \in \mathcal{A}} P\left(X_{a}\right) P\left(Y_{b} \mid X_{a}\right) \log \frac{P\left(Y_{b} \mid X_{a}\right)}{P\left(Y_{b}\right)}
$$

where $P\left(Y_{b}\right)=\sum_{a \in \mathcal{A}} P\left(X_{a}\right) P\left(Y_{b} \mid X_{a}\right), \forall b \in \mathcal{A}$. The channel capacity is given by maximizing the mutual information between $X$ and $Y$ with respect to the input probability distribution, i.e. $C=\max _{P\left(X_{a}\right)} I(X ; Y)$, where $P\left(X_{a}\right)$ is the a-priori probabilities.



Fig. 11. Optimum a-priori probabilities for systems using original and modified TX-B.

As we have stated in the previous section, the channel input $X$ has a uniform distribution in all the previous simulations, $P\left(X_{a}\right)=1 / 4$ for $\forall a \in \mathcal{A}$. With this uniform $P\left(X_{a}\right)$, the mutual information calculated from (12) will not in general be maximized because $P\left(Y_{b} \mid X_{a}\right)$ is not symmetric, which is indicated by the signal constellations given in Figures 6 and 7 . Thus, we first modify the input a-priori probabilities $P\left(X_{a}\right)$ so that the maximum mutual information can be achieved. To find the optimum $P\left(X_{a}\right)$, the MATLAB function fminsearch is used. Given initial probabilities of $P\left(X_{a}\right)$, this function returns the optimum $P\left(X_{a}\right)$ that gives the local maximum of $I(X, Y)$. Although it is not proved that the mutual information for the studied channel is a concave function of $P\left(X_{a}\right)^{3}$, by trying different initial values of $P\left(X_{a}\right)$, the same output results imply that the local maximum is also the global maximum. The obtained $I(X ; Y)$ is thus the channel capacity. The reason that $P\left(Y_{b} \mid X_{a}\right), \forall a, b \in \mathcal{A}$ is not independent of $P\left(X_{a}\right)$ is because $P^{a}(i)$ in (10) is a function of $P\left(X_{a}\right)$, i.e. the probability of each point in the symbol clouds is a combination of $a$ priori probabilities of $m+1$ symbols. Also for this reason, the numerical Blahut-Arimoto algorithm [35, pp. 366-367] is not applicable in its standard form.

In Fig 11, the optimum a-priori probabilities versus $E_{b} / N_{0}$ are plotted. The figure clearly shows that the optimum input distribution is uniform only at high $E_{b} / N_{0}$. We compare the mutual information with the optimum and uniform input distribution in Fig 12. As it shows, the difference between the two mutual information is negligible. We therefore conclude that the modification of using optimum a-priori probabilities $P\left(X_{a}\right)$ is not efficient in improving the channel mutual information for this particular type of channel.

From our previous study of a coherent QPSK system in [1], we know that a transmitter modification will change the signal constellation significantly, which means that the channel

\footnotetext{
${ }^{3} P\left(Y_{b} \mid X_{a}\right), \forall a, b \in \mathcal{A}$ is not independent of $P\left(X_{a}\right)$ in the studied channel. The convexity of mutual information proved in [35, p. 31$]$ is for the case that $P\left(X_{a}\right)$ and $P\left(Y_{b} \mid X_{a}\right)$ are independent.
} 


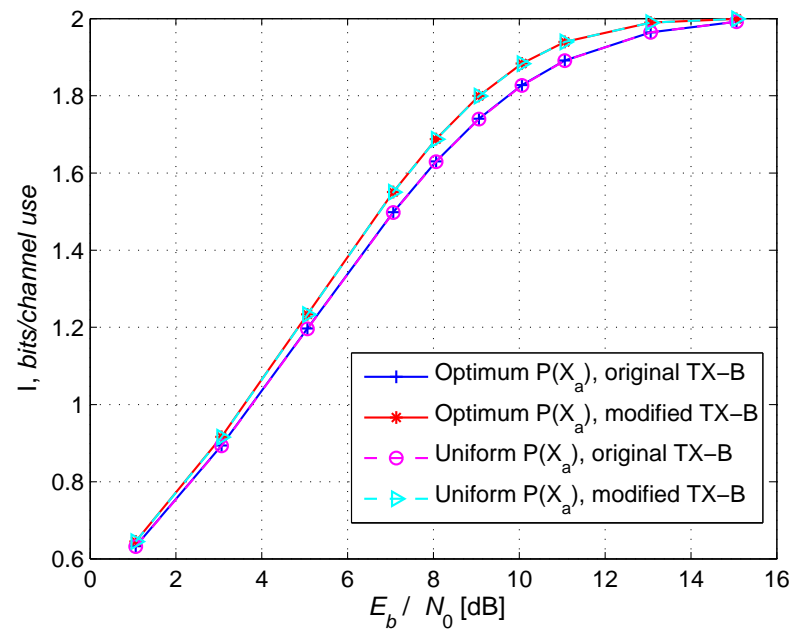

Fig. 12. Mutual information with different input distribution for systems using original and modified TX-B .

TABLE I

COEFFICIENTS OF TRANSMITTER MAPPING UNIT.

\begin{tabular}{c|cccccccc}
\hline \hline $\begin{array}{c}E_{b} / N_{0} \\
{[\mathrm{~dB}]}\end{array}$ & 1 & 3 & 5 & 7 & 9 & 11 & 13 & 15 \\
\hline$\lambda$ & 1.05 & 1.05 & 1.06 & 1.06 & 1.06 & 1.06 & 1.06 & 1.07 \\
$\mu$ & 1.05 & 1.05 & 1.05 & 1.05 & 1.05 & 1.05 & 1.05 & 1.05 \\
\hline \hline
\end{tabular}

transition probabilities will be changed accordingly and the channel mutual information will undoubtedly be affected. Here, a similar transmitter modification as in [1] is applied for a system using TX-B. The aim of this modification is to change signal constellations by modifying the transmitted signal. The transmitted signal in (2) is modified as

$$
f_{B}(t)=\eta \cdot e^{j \frac{\pi}{2} \sum_{n}\left(\lambda a_{n}+\mu b_{n}\right) p(t-n T)},
$$

where the two coefficients of the transmitter mapping unit, $\lambda$ and $\mu$, together with the a-priori probabilities $P\left(X_{a}\right)$ are the parameters that need to be optimized with respect to maximizing the channel mutual information. Again, we use MATLAB function fminsearch to obtain the optimum $\lambda, \mu$ and $P\left(X_{a}\right)$, and with that the local maximum $I(X, Y)$ is obtained. By trying different initial $P\left(X_{a}\right)$ and reasonable $\lambda$ and $\mu$, the same search results indicate that the local maximum obtained is the global maximum, and is the capacity. In Table I, the optimum coefficients $\lambda$ and $\mu$ are listed for different SNRs when the system uses TX-B. To compare with the system using original transmitter, we plot the optimum $P\left(X_{a}\right)$ and the channel mutual information for systems using modified TX-B also in Figures 11 and 12, respectively. The capacity improvement with the modified TX-B is about $0.9 \mathrm{~dB}$ at a target of 1.9 bits/channel use, while it becomes greater when the targeting capacity is close to 2 bits/channel use. Again, the difference between two channel mutual information for systems using a modified TX-B is negligible, which indicates that the effects of input distribution on the channel mutual information can be ignored.
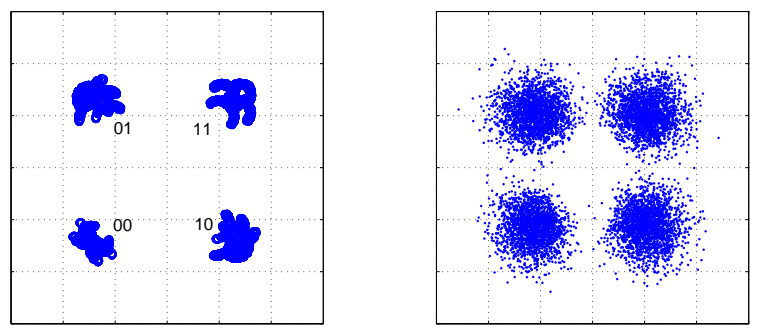

Fig. 13. Simulated signal constellation $\left(Y_{I}, Y_{Q}\right)$ without (left) and with (right) noise when using the modified TX-B, $E_{b} / N_{0}=13 \mathrm{~dB}$ when with noise.

\section{SYSTEM MODIFICATION AND RESULTS}

The previous section demonstrates the channel capacity improvement with the transmitter modification and shows that the effect of the input distribution on the capacity can be ignored. In this section, with a uniform distributed input, the transmitter modification is evaluated in terms of SER and BER performances for systems using TX-A and TX-B. Note that this modification requires no hardware add-on and the system complexity is not increased.

In (13), the modified transmitted signal for a system using TX-B is given. For a system using TX-A, the transmitted signal is modified as

$$
f_{A}(t)=\eta \cdot \sin \left[\frac{\pi}{2} \sum_{n}\left(\lambda a_{n} p(t-n T)\right)\right] e^{j \frac{\pi}{2} \sum_{n} \mu b_{n} p(t-n T)} .
$$

Again, $\mu$ and $\lambda$ can be found by maximizing mutual information $I(X, Y)$.

To illustrate the change in the signal constellation, we plot the signal constellation for a system using a modified TX-B in Fig. 13. A comparison with Fig. 7 shows that the modified signal constellation is much more symmetric. In particular, the clouds of symbol 11 are less dislocated, which will give a better SER performance and consequently a better overall system BER performance.

In Figures 14 and 15, the simulated SER performance for systems using modified TX-A and TX-B are compared with the systems using the original transmitters, respectively. Since the system with TX-B has a more asymmetric signal constellation, the modification, which gives a much more symmetric signal constellation, achieves a greater improvement than the system using TX-A. It should be noted that although the optimum $\lambda$ and $\mu$ vary with the SNR as shown in Table I, for convenience we use the same $\lambda$ and $\mu$ for all SNRs in the simulations, which implies that the improvement can be even greater if the optimum $\lambda$ and $\mu$ are applied.

Fig. 16 shows the BER improvements with the modified systems. For systems using TX-A, this improvement is about $1 \mathrm{~dB}$ at a BER target of $10^{-6}$ and the curve trend shows that a higher gain can be achieved at a lower BER. For the system using TX-B, the improvement is more than $3 \mathrm{~dB}$ at a BER of $10^{-6}$, and we believe that it will be even greater if the targeting BER is set at $10^{-9}$, the commonly required BER for fiber-optic systems. 


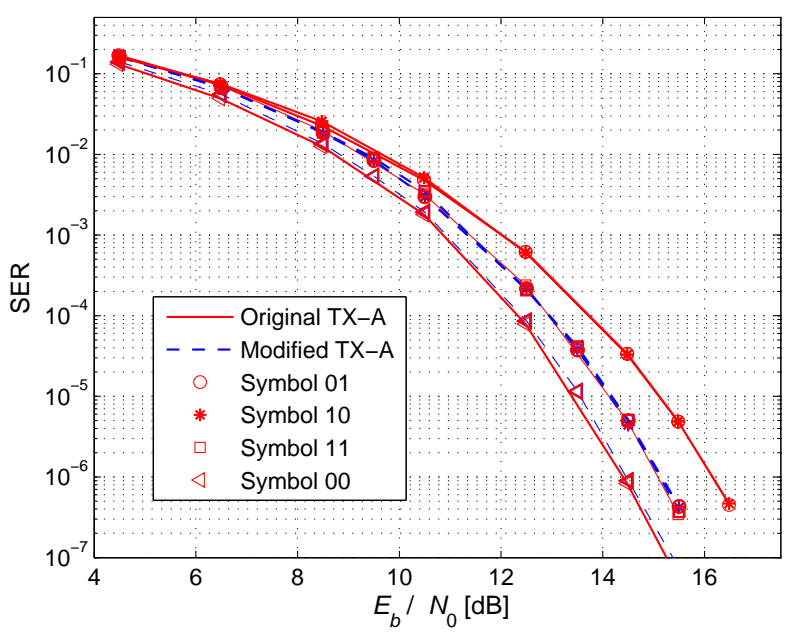

Fig. 14. Comparison of the simulated SERs for systems using an original and a modified TX-A, $\lambda=1.04, \mu=1.06$.



Fig. 15. Comparison of the simulated SERs for systems using an original and a modified TX-B, $\lambda=1.06, \mu=1.05$.



Fig. 16. Comparison of the average BERs.

\section{DISCUSSION AND CONCLUSIONS}

In this paper, we present a novel and unique property of a differential QPSK fiber-optic system, i.e., the four DQPSK symbols have significantly different performances. We found by analysis and numerical simulations that the performance difference is about $2 \mathrm{~dB}$ and $4 \mathrm{~dB}$ targeting at a symbol error rate of $10^{-6}$ when using phase modulator based transmitters, TX-A and TX-B in this paper, respectively. This unequal SER phenomenon has its source in the combined ISI of the electro-optic modulator used in the transmitter and the lowpass filtering used in the receiver. This combined ISI is unique for fiber-optic channels due to the imperfect transmitters and receivers and the practical difficulty of using a matched filter. This ISI causes an unequal bit error rate in a coherent QPSK system [1], but only causes symbol error rates different in a DQPSK system with the average BER of the two symbol bits remaining the same. Since most experimental DQPSK studies use only a single differential detector, detecting one quadrature at a time, the SER is seldom discussed and the unequal SER phenomenon has been unnoticed. By using the developed system model in this paper, the symbol error rates are derived for the first time. The unequal SER phenomenon is reported and explained.

Based on the analytic channel model, we compute the channel mutual information and propose two modifications to improve it. The more efficient alternative is to modify the configuration of the transmitter, which requires no hardware add-on and thus no increase on the system complexity. This transmitter modification improves the system capacity by up to $0.9 \mathrm{~dB}$ when using TX-B. With this proposed modification, the system performance in terms of BER is improved by $1 \mathrm{~dB}$ and $3 \mathrm{~dB}$ respectively at a target of $10^{-6}$ when using TX-A and TX-B, and this improvement will be even greater when the target BER is set at $10^{-9}$, the common required BER for fiberoptic systems. The results in this paper also emphasize the importance of considering not only the constellation diagram of the system, but also the paths between the symbols, to properly account for the ISI. We hope that this finding will be valuable when designing multilevel differential systems. In particular, we believe that special attention should be paid to the unequal symbol performance in the code design for coded systems.

\section{REFERENCES}

[1] H. Zhao, E. Agrell, and M. Karlsson, "Unequal bit error probability in coherent QPSK fiber-optic systems using phase modulator based transmitters," European Transactions on Telecommunications, vol. 19, no. 8, pp. 895-906, Dec. 2008.

[2] A. H. Gnauck, R. W. Tkach, A. R. Chraplyvy, and T. Li, "High-capacity optical transmission systems," IEEE Journal of Lightwave Technology, vol. 26, no. 9, pp. 1032-1045, May 2008.

[3] J. G. Proakis, Digital Communications, McGraw-Hill, 4 ed., 2001.

[4] G. Kramer, A. Ashikhmin, A. J. van Wijngaarden, and X. Wei, "Spectral efficiency of coded phase-shift keying for fiber-optic communication," IEEE Journal of Lightwave Technology, vol. 21, no. 10, pp. 2438-2445, Oct. 2003.

[5] J. M. Kahn and K.-P. Ho, "Spectral efficiency limits and modulation/detection techniques for DWDM systems," IEEE Journal of Selected Topics in Quantum Electronics, vol. 10, no. 2, pp. 259-272, Mar-Apr. 2004. 
[6] C. Wree, N. Hecker-Denschlag, E. Gottwald, P. Krummrich, J. Leibrich, E.-D. Schmidt, B. Lankl, and W. Rosenkranz, "High spectral efficiency $1.6 \mathrm{~b} / \mathrm{s} / \mathrm{Hz}$ transmission $(8 \times 40 \mathrm{~Gb} / \mathrm{s}$ with a $25-\mathrm{GHz}$ grid) over $200-$ km SSMF using RZ-DQPSK and polarization multiplexing," IEEE Photonics Technology Letters, vol. 15, no. 9, pp. 1303-1305, Sept. 2003.

[7] S. Ferber, C. Schubert, R. Ludwig, C. Boerner, C. Schmidt-Langhorst, and H. G. Weber, "640 Gbit/s DQPSK, single wavelength-channel transmission over $480 \mathrm{~km}$ fibre link," Electronics Letters, vol. 41, no. 22, pp. 1236-1237, Oct. 2005.

[8] P. S. Cho, G. Harston, C. J. Kerr, A. S. Greenblatt, A. Kaplan, Y. Achiam, G. Levy-Yurista, M. Margalit, Y. Gross, and J. B. Khurgin, "Investigation of 2-b/s/Hz 40-Gb/s DWDM transmission over 4 x 100 km SMF-28 fiber using RZ-DQPSK and polarization multiplexing," IEEE Photonics Technology Letters, vol. 16, no. 2, pp. 656-658, Feb. 2004.

[9] N. Yoshikane and I. Morita, " $1.14 \mathrm{~b} / \mathrm{s} / \mathrm{Hz}$ spectrally efficient 50 x 85.4$\mathrm{Gb} / \mathrm{s}$ transmission over $300 \mathrm{~km}$ using copolarized RZ-DQPSK signals," IEEE Journal of Lightwave Technology, vol. 23, no. 1, pp. 108-114, Jan. 2005.

[10] S. Chandrasekhar, X. Liu, D. Kilper, C. R. Doerr, A. H. Gnauck, E. C. Burrows, and L. L. Buhl, "Terabit transmission at $42.7-\mathrm{Gb} / \mathrm{s}$ on $50-\mathrm{GHz}$ grid using hybrid RZ-DQPSK and NRZ-DBPSK formats over 16 × 80 km SSMF spans and 4 bandwidth-managed ROADMs," IEEE Journal of Lightwave Technology, vol. 26, no. 1, pp. 85-90, Jan. 2008.

[11] T. Ito, S. Fujita, E. L. T. de Gabory, S. Shioiri, and K. Fukuchi, "Precise analysis of transmission impairments of Pol-Mux 110Gb/s RZDQPSK with automatic Pol-Dmux using straight 2,000-km SMF line," in Proc. European Conference and Exhibition on Optical Communication (ECOC), Brussel, Belgium, Sept. 2008.

[12] M. P. Fedoruk, S. K. Turitsyn, O. V. Shtyrina, M. V. Zakharyuta, A. V. Yakasov, A. Shafarenko, S. R. Desbruslais, K. Reynolds, and R. Webb, "Patterning of errors in $40 \mathrm{Gbit} / \mathrm{s}$ WDM RZ-DBPSK SMF/DCF optical transmission system," in Proc. Optical Fiber Communication Conference (OFC), Anaheim, USA, Mar. 2007.

[13] T. Wuth, E. Agrell, M. Karlsson, and M. Sköld, "Fiber communications using convolutional coding and bandwidth-efficient modulation," Optics Express, vol. 14, no. 2, pp. 542-555, Jan. 2006.

[14] J. Wang and J. M. Kahn, "Conventional DPSK versus symmetrical DPSK: Comparison of dispersion tolerances," IEEE Photonics Technology Letters, vol. 16, no. 6, pp. 1585-1587, June 2004.

[15] M. Cavallari, C. R. S. Fludger, and P. J. Anslow, "Electronic signal processing for differential phase modulation formats," in Proc. Optical Fiber Communication Conference (OFC), Los Angeles, USA, Feb. 2004

[16] J. Wang and J. M. Kahn, "Performance of electrical equalizers in optically amplified OOK and DPSK systems," IEEE Photonics Technology Letters, vol. 16, no. 5, pp. 1397-1399, May 2004.

[17] G. Bosco, V. Curri, E. Roffe, and P. Poggiolini, "Joint effect of MLSE and receiver filters optimization on dispersion robustness of IMDD, DPSK, DQPSK, and duobinary modulation," IEEE Photonics Technology Letters, vol. 19, no. 24, pp. 2003-2005, Dec. 2007.

[18] G. P. Agrawal, Fiber-Optic Communication Systems, New York, USA: John Wiley \& Sons, Inc., 2002.

[19] M. Ohm and T. Freckmann, "Comparison of different DQPSK transmitters with NRZ and RZ impulse shaping," in Proc. IEEE/LEOS Workshop on Advanced Modulation Formats, San Francisco, USA, July 2004.

[20] M. Serbay, C. Wree, and W. Rosenkranz, "Comparison of six different RZ-DQPSK transmitter set-ups regarding their tolerance towards fibre impairments in $8 \times 40 \mathrm{~Gb} / \mathrm{s}$ WDM-systems," in Proc. IEEE/LEOS Workshop on Advanced Modulation Formats, San Francisco, USA, July 2004.

[21] H. Zhao, E. Agrell, and M. Karlsson, "Transmitter comparison and unequal bit error probabilities in coherent QPSK systems," in Proc. Optical Fiber Communication Conference (OFC), Anaheim, USA, Mar. 2007.

[22] X. Wu, L. Christen, S. R. Nuccio, O. F. Yilmaz, L. Paraschis, Y. K. Lize, and A. E. Willner, "Experimental synchronization monitoring of I/Q data and pulse-carving temporal misalignment for a serial-type 80Gbit/s RZ-DQPSK transmitter," in Proc. Optical Fiber Communication Conference (OFC), San Diego, USA, Feb. 2008.

[23] P. J. Winzer, M. Pfennigbauer, M. M. Strasser, and W. R. Leeb, "Optimum filter bandwidths for optically preamplified NRZ receivers," IEEE Journal of Lightwave Technology, vol. 19, no. 9, pp. 1263-1273, Sept. 2001.

[24] M. Serbay, C. Wree, and W. Rosenkranz, "Implementation of differential procoder for high-speed optical DQPSK transmission," Electronics Letters, vol. 40, no. 21, pp. 1288-1289, Sept. 2004.

[25] O. E. Agazzi, M. R. Hueda, H. S. Carrer, and D. E. Crivelli, "Maximumlikelihood sequence estimation in dispersive optical channels," IEEE
Journal of Lightwave Technology, vol. 23, no. 2, pp. 749-763, Feb. 2005.

[26] C. Xia and W. Rosenkranz, "Nonlinear electrical equaliztion for different modulation formats with optical filtering," IEEE Journal of Lightwave Technology, vol. 25, no. 4, pp. 996-1001, Apr. 2007.

[27] V. Curri, R. Gaudino, A. Napoli, and P. Poggiolini, "Electronic equalization for advanced modulation formats in dispersion-limited systems," IEEE Photonics Technology Letters, vol. 16, no. 11, pp. 2556-2558, Nov. 2004.

[28] R. A. Griffin and A. C. Carter, "Optical differential quadrature phaseshift key (oDQPSK) for high capacity optical transmission," in Proc. Optical Fiber Communication Conference (OFC), Anaheim, USA, Mar. 2002.

[29] M. Pfennigbauer, M. M. Strasser, M. Pauer, and P. J. Winzer, "Dependence of optically preamplified receiver sensitivity on optical and electrical filter bandwidths-measurement and simulation," IEEE Photonics Technology Letters, vol. 14, no. 6, pp. 831-833, June 2002.

[30] C. Wang and J. Yan, "Filter design for noncoherent receivers," Master Thesis EX023/2006, Department of Signals and Systems, Chalmers University of Technology, Göteborg, Sweden, 2006.

[31] J. D. Downie, I. Tomkos, N. Antoniades, and A. Boskovic, "Effects of filter concatenation for directly modulated transmission lasers at 2.5 and $10 \mathrm{~Gb} / \mathrm{s}, "$ IEEE Journal of Lightwave Technology, vol. 20, no. 2, pp. 218-228, Feb. 2002.

[32] P. J. Winzer, S. Chandrasekhar, and H. Kim, "Impact of filtering on RZ-DPSK reception," IEEE Photonics Technology Letters, vol. 15, no. 6, pp. 840-842, June 2003.

[33] S. R. Chinn, D. M. Boroson, and J. C. Livas, "Sensitivity of optically preamplified DPSK receivers with Fabry-Perot filters," IEEE Journal of Lightwave Technology, vol. 23, no. 1, pp. 370-376, Mar. 2005.

[34] X. Zhang, G. Zhang, C. Xie, and L. Wang, "Noise statistics in optically preamplified differential phase-shift keying receivers with MachZehnder interferometer demodulation," Optics Letters, vol. 29, no. 4, pp. 337-339, Feb. 2004.

[35] T. M. Cover and J. A. Thomas, Elements of Information Theory, John Wiley \& Sons, Inc., 1991. 